\title{
The Resolution of the Great 20th Century Debate in the Foundations of Mathematics
}

\author{
Edgar E. Escultura \\ GVP-Professor V. Lakshmikantham Institute for Advanced Studies, GVP College of Engineering, JNT University \\ Kakinada Madurawada Visakhapatnam, India \\ Email: escultur36@gmail.com
}

Received 30 November 2015; accepted 21 February 2016; published 26 February 2016

Copyright (C) 2016 by author and Scientific Research Publishing Inc.

This work is licensed under the Creative Commons Attribution International License (CC BY). http://creativecommons.org/licenses/by/4.0/

(c) (i) Open Access

\section{Abstract}

The paper resolves the great debate of the $20^{\text {th }}$ century between the three philosophies of mathematics-logicism, intuitionism and formalism-founded by Bertrand Russell and A. N. Whitehead, L. E. J. Brouwer and David Hilbert, respectively. The issue: which one provides firm foundations for mathematics? None of them won the debate. We make a critique of each, consolidate their contributions, rectify their weakness and add our own to resolve the debate. The resolution forms the new foundations of mathematics. Then we apply the new foundations to assess the status of Hilbert's 23 problems most of which in foundations and find out which ones have been solved, which ones have flawed solutions that we rectify and which ones are open problems. Problem 6 of Hilbert's problems-Can physics be axiomatized?-is answered yes in E. E. Escultura, Nonlinear Analysis, A-Series: 69(2008), which provides the solution, namely, the grand unified theory (GUT). We also point to the resolution of the 379-year-old Fermat's conjecture (popularly known as Fermat's last theorem) in E. E. Escultura, Exact Solutions of Fermat's Equations (Definitive Resolution of Fermat's Last Theorem), Nonlinear Studies, 5(2), (1998). Likewise, the proof of the 274-year-old Goldbach's conjecture is in E. E. Escultura, The New Mathematics and Physics, Applied Mathematics and Computation, 138(1), 2003.

\section{Keywords}

Axiom of Choice, Banach-Tarski Paradox, Goldbach's Conjecture, Logicism, Constructivism, Fermat's Conjecture, Field Axioms, Formalism, Qualitative Modelling, Rational Thought, Self-Reference

\section{Introduction}

Technical mathematics — the mathematics in the various specialized fields of mathematics—has expanded tre- 
mendously, especially, nonlinear analysis but work on the foundations of mathematics has stopped by the latter half of the $20^{\text {th }}$ Century. Although the only living founder of logicism and pioneer in foundations, Bertrand Russell, was still active in the 1960s, he was consumed by his opposition to nuclear weapons and the Vietnam War during which time he founded and convened the Bertrand Russell Peace Foundation which tried the United States for crimes against humanity in Vietnam (he passed away in 1970 at the age of 97). We revisit the foundations of mathematics and resolve the great debate of the $20^{\text {th }}$ Century among logicism [1], intuitionism [2] and formalism [3] founded, respectively, by Bertrand Russell (1872-1970) and A. N. Whitehead (1861-1947) [4] [5], L. E. J. Brouwer (1881-1966) [6] and David Hilbert (1862-1946) [7]. The issue: which one provides firm foundations for mathematics [8]? None of them won the debate. Therefore, we consolidate their contributions and add our own to resolve the debate and call the resolution the new foundations of mathematics. We apply the new foundations to David Hilbert's 23 problems of mathematics [9] most of which problems in foundations and assess which ones are solved, which solutions are flawed in which case we rectify them and which ones are open problems. Problem 6-Can physics be axiomatized?-is answered yes in 2008 and the solution is The Grand Unified Theory [10]. We solve other long standing unsolved problems including the 379-year-old Fermat's conjecture (also known as Fermat's last theorem (FLT) [11] and the 274-year-old Goldbach's conjecture [12]. The paper summarizes the requirements for avoiding uncontained ambiguity and paradox or contradiction.

We introduce qualitative mathematics, the complement of computation and measurement and qualitative model of rational thought [13], part of the main contribution of [14], consisting of this activity:

Making conclusions, visualizing, abstracting, thought experimenting, learning, creating mathematical spaces, intuition, imagination, trial and error to sift out what is correct, negating what is known to gain insights into the unknown, altering premises to draw out new conclusions, thinking backwards and devising strategies and techniques that yield results.

\section{The Great 20th Century Debate}

Why is the resolution of this great debate important? Important mainly because technical mathematics cannot solve problems in foundations and most long standing unsolved problems, e.g., FLT and Goldbach's conjecture, including most of Hilbert's 23 problems, belong to this category. To resolve the issue of contention in the debate we look at the sources of ambiguity and paradox or contradiction.

\subsection{Sources of Ambiguity}

Ambiguity erodes the foundations of mathematics. Therefore, we identify the sources of ambiguity. Ambiguity and contradiction are intertwined because when there is ambiguity in a mathematical space one can find contradictory statements or a counterexample (which is a contradiction) as shown by the work of Imre Lakatos [15] where he found a counterexample at every step of Cauchy's proof of Euler's formula on the relationship between the vertices, edges and faces of a polyhedron including his own remedy to the contradiction. We find ways to avoid them.

\subsubsection{Vacuous Concept}

One such source of contradiction is vacuous (ill-defined or non-existent) concept. Consider the concept, the root of the equation $x^{2}+1=0$ (among the real numbers) denoted by $i=\sqrt{-1}$. Since the equation has no root the concept $i$ is vacuous, does not exist. Consequently,

(1) $i=\sqrt{-1}=\sqrt{1 /-1}=1 / i=-i$, from which follows $1=0$ and $i=0$ which collapse both the real and complex number systems. The concept is vacuous for another reason: the mapping $\sqrt{a}$ is defined only on non-negative real number $a$ where $a$ is a perfect square. In fact, the nth root of $a$, i.e., $\sqrt[n]{a}$ is defined only if $a$ is a perfect nth power of some real number.

Another contradiction arising from a vacuous concept called Perron Paradox [11] follows:

Let $N$ be the largest integer. Since the integers are linearly ordered by the relation $<$, one and only one of the following holds: $N<1, N=1,1<N$. The first inequality is out, so is the second since $N<N^{2}$ and $N$ would not be the greatest integer. Therefore, $N=1$ which is false.

The culprit: $N$ is vacuous, does not exist.

The concept vacuousity applies to vacuous propositions and problems. This is the reason a careful mathematician pays particular attention to a suitable theorem on the existence of a solution of a problem before actually 
solving the problem. The constructivist requires not only existence but also an algorithm for computing the solution. That algorithm makes sense only when there is a solution.

\subsubsection{Infinity}

In traditional mathematics a set is infinite if it can be put into one-one-correspondence with a proper subset. We modify this definition by identifying the concept infinity with its essential property of inexhaustibility. We take inexhaustibility as the defining quality of infinity and this clearly includes the traditional definition. For example, if we count the digits of a nonterminating decimal and label the digits we have already counted by, say, $x_{1}, x_{2}, \cdots, x_{n}$, then a sequence, $x_{1}, x_{2}, \cdots, x_{n}, \cdots, n=1,2, \cdots$, is generated that has no last element and the counting is never complete. This is an ambiguity. It is not the case with a terminating decimal where there is a last element so that its digits are finite. This is an example of countable infinity denoted by $\infty$. It is a concept that pervades mathematics and the only type of infinity that exists as we shall see later. It is neither a real number nor a counting number and, naturally, the binary additive and multiplicative operations do not apply to $\infty$ and if real numbers are added to a given real number one at a time $\infty$ can never be reached. Thus, there is no boundary between the real numbers and infinity that can be crossed. In other words, none of the binary operations in the real number system applies to it.

Since the binary operations addition and multiplication are defined only on terminating decimals the nonterminating decimals are ill-defined and, therefore, ambiguous in the real number system. Therefore, any concept defined in terms of nonterminating decimal is ambiguous, ill-defined. An example of such ambiguous concept is irrational number (rational for short) defined as nonperiodic nonterminating decimal. Furthermore, periodicity or non-periodicity of a nonterminating decimal is not verifiable. Thus, an irrational number has at least two layers of ambiguity. This ambiguity is illustrated by the fact that the sum of $\sqrt{3}$ and $\sqrt{2}$ is unknown. Moreover, not every rational numbers (rationals for short), i.e., the quotients of two integers, is a real number; only when the divisor has no prime factor other than 2 and 5 is the quotient a terminating decimal, a rational and a real number. Thus, the rationals coincide with the terminating decimals and they are the only defined real numbers.

In the new real number system $\mathbf{R}^{*}$ of which the real number system $\mathbf{R}$ is a subspace, the concept nonterminating decimal is defined for the first time [16] [17] but has contained ambiguity. Ambiguity of a concept is contained if it is defined, i.e., it exists, and is approximated with a desired error by a defined concept. As example, the infinite set of decimal digits of a nonterminating decimal is contained and its ambiguity is likewise contained since if we cut or truncate a nonterminating decimal at the $\mathrm{n}^{\text {th }}$ decimal digit, the margin or fraction of error is less than $10^{-\mathrm{n}}$. Here, the truncated number is a terminating decimal which is defined in the real number system. The error is the upper bound of the value or norm of the discarded tail of the nonterminating decimal in the truncation. The maximum error occurs when the discarded digits are all $9 \mathrm{~s}$. In a mathematical space this type of ambiguity can be contained or isolated and does not cause a problem; in fact, containing an ambiguity or reducing an error to a desired number is a standard technique in approximation theory. Therefore, it is admissible. Even the definition of a rational (rational for short) as quotient of two integers is ambiguous. In fact, division of an integer by a prime other than 2 and 5 is ambiguous since the quotient is a nonterminating decimal. What is correct is that the rationals coincide with the terminating decimals. Even the alternative definition of a rational as periodic decimal is incorrect. The periodic decimal $0.99 \ldots$ is counterexample to it since neither addition nor multiplication is defined on it.

Ambiguity of a concept means that not all of its properties can be verified. It follows that any proposition including axiom or theorem involving an ambiguous concept is ambiguous, i.e., its conclusion is uncertain. In particular, a proposition involving the universal quantifier every, or the existential quantifier there exists applied to an infinite set is ambiguous due to the inexhaustibility of the latter. For example, suppose we want to prove that "every element of an infinite set S has property P". Start with an element $x_{1}$ and suppose it has this property (otherwise, the statement is outright false), then take another element $x_{2}$ and check if it has this property, etc. Then since $\mathrm{S}$ is inexhaustible verification of the truth of this statement is never complete, i.e., the statement is ambiguous. Similarly, by the same algorithm but starting with an element that does not have this property, it may not be possible to prove that there exists an element of $S$ that has this property which is an ambiguity. Every infinite mathematical space is presently tainted with this type of ambiguity from the definition of limit of real analysis through the field axioms of the real number system [16]. Thus, the real number system is presently ambiguous. Consequently, FLT being formulated in it is also ambiguous and has no proof. It was this ambiguity of the real number system that catalyzed its critique-rectification as the new real number system [15] [16], the 
reformulation of FLT in it and its resolution in 1998 that proved it false [18]. In some cases, it may not be possible to choose a particular element $x_{1}$ of an infinite set, e.g., in the construction of a non-measurable set the axiom of choice was invoked [19] [20]. This axiom is flawed when applied to infinite set because of the ambiguity of the latter.

Consider the axiom of choice, one of the axioms of the Zermelo-Fraenkel set theory or ZFC that says [20].

Given any set of mutually disjoint nonempty family of sets there exists at least one set that contains exactly one element in common with each of the nonempty sets.

The elements of the set are mapped one-one into the family of mutually disjoint sets and the mapping is called the choice function.

This seems obvious but it is tainted with ambiguity by the existential quantifier when applied to infinite set. The axiom of choice or its variants - the completeness axiom and Dedekind cut-is involved everywhere in analysis, e.g., as one of the field axioms of the real number system and in proving limits (which involves the existential and universal quantifiers) and existence of a nonmeasurable set [11]. Moreover, there is no flawless proof of existence of non-measurable set. In other words, measurability is not an issue at this time. Therefore, real analysis can be simplified by dropping the requirement of measurability in analysis.

Consider Cantor's diagonal method [21] [22] in proving that the real numbers form a nondenumerable set. This method is flawed by the application of the existential quantifier "there exists" on infinite set. Moreover, the set of off diagonal elements generated by Cantor's diagonal method is a countable union of countable sets which is countable. Furthermore, it uses the indirect proof which is self-referent and ambiguous. Therefore, Cantor's diagonal method is flawed. With the failure of Cantor's diagonal method, the continuum hypothesis [23] [24] also collapses. This resolves problem 1 of Hilbert's 23 problems that seeks a transfinite number between a denumerable set and the continuum. The problem is vacuous and has no solution since the continuum in the sense of Cantor does not exist aside from the use of the indirect proof and the axiom of choice in the proof.

A recent surprise is the Banach-Tarski paradox [25] which has been used as a counterexample to the axiom of choice. First of all, the axiom of choice is ambiguous on its own terms when applied to infinite set and does not need a counterexample to confirm it.

The Banach-Tarski paradox is one of the most celebrated paradoxes in mathematics. One version of it states that given any two subsets $A$ and $B$ of $\boldsymbol{R}^{3}$, which are bounded and have non-empty interior, it is possible to 'cut' A into a finite number of pieces which can be moved by rigid motions (translations and rotations) to form exactly $B$. This has many amusing consequences; it is most commonly stated as the assertion that a baseball can be cut into a finite number of pieces which can then be reassembled without distortion to form a ball as big as the Earth. The proof is in [25]; we note the following important points about it:

(1) In the proof of Proposition 3.1, Section 3, p. 4, which is part of the proof of the paradox, is the expression,

$$
\left(1 / 3^{n}\right)(a \sqrt{2}, b, c \sqrt{2}) \text {, }
$$

involving division of an integer by a prime other than 2 and 5; both the quotient and the product are ill-defined because they involve decimals which are also ill-defined. Therefore, the quotient $1 / 3^{\mathrm{n}}$ does not exist and, naturally, the product in (1) does not exist. Ill-defined concepts appear many times in the proof.

(2) On page 8, the paragraph after fact (3), part of the sentence that begins in line 2 says:

“....and there are uncountably many lines through the origin in $\boldsymbol{R}^{3,}$

which is, again, vacuous since those lines do not exist?

Only one of items (1) and (2) suffices to show that the Banach-Tarski paradox does not exist.

The ambiguity of infinity has far reaching implications for mathematics. It affects theorems in infinite mathematical spaces. It limits the domain of the real number system to terminating decimals, i.e., a nonterminating decimal is ill-defined (ambiguous). Thus, the concept irrational as nonperiodic nonterminating decimal is illdefined and ambiguous since it is unverifiable. This is the reason it is impossible to add $\sqrt{2}$ and $\sqrt{3}$ since both terms are ambiguous. The rationals as quotients are limited to terminating decimals because division by a prime number other than 2 and 5 is ill-defined the quotient being nonterminating.

Moreover, ambiguity or uncertainty applies to small and large numbers. For example, changing a small divisor less than $1(<1)$ leaves a wide margin of error. Furthermore, the digits of both small and large numbers (depending on context) cannot be entered into the calculator or computer screen and this is an ambiguity. In dealing with a large number the scientific notation is used which approximates the number by the largest order of magnitude and discarding the digits and terms of lower orders of magnitude. For a small number the nonzero (de- 
cimal) digits are too far to the right in the decimal expansion that it is difficult to distinguish its location relative to 0 . This uncertainty applies to tiny object at great speed, e.g., electron at speed close to that of light which accounts for the Heisenberg's uncertainty principle of physics [26]. This is an example of motion in the real world affected by number not because of the numbers involved but the difficulty in determining the position of a tiny object and measuring large numbers. Furthermore, large integers have different behavior described by the law of large numbers in statistics. For instance, it is noted in [27] that the behavior of an integer $\mathrm{N}$ abruptly changes when its value goes past $10^{k}$, where $k=10^{70}$. Thus, both extremes of the norm of a real number suffer from ambiguity.

\subsubsection{Russell Paradoxes}

In 1901 Bertrand Russell who was then working on the Principles of Mathematics, rocked set theorists and logicians with this discovery named after him (Russell antimony) [28]:

Let $M$ be the set of all sets where each element does not belong to itself, i.e., $M=\{m: m \notin m\}$. Either $M \in M$ or $M \notin M$. If $M \notin M$, its defining conditions hold; therefore $M \in M$. On the other hand, if $M \in M$, then $M$ also satisfies its defining condition; therefore $M \in M$ and $M \notin M$.

Russell sent it in a letter to G. Frege in 1902 [28] just as Frege had submitted Grundlagen der Arithmetik that, like the Principles of Mathematics, attempted to build arithmetic on set theory, the foundation of logicism. The letter invalidated much of the rigor of the work and Frege was forced to add a note at the end stating, "A scientist can hardly meet with anything more undesirable than to have the foundation give way just as the work is finished. I was put in this position by a letter from Mr. Bertrand Russell when the work was nearly through the press" [28].

A contradiction arises whenever the expression "the set of all sets" is involved for it leads to the concept of a set being a member of itself and, therefore, not a member of itself. In other words, the universal set and power set do not exist, they are vacuous. This, again, is an example, of thought creating nonsense. There are, in fact, numerous Russell paradoxes that can be reduced to this type of paradox [29]; we give another example, the barber paradox [30]:

The barber of Seville shaves those who do not shave themselves. Who shaves the barber?

If the barber shaves himself then he does not shave himself; if he does not then he shaves himself. Obviously, there is no correct answer here, i.e., the barber of Seville does not exist. Thus, a Russell paradox reduces to a vacuous proposition.

\subsubsection{Self-Reference}

If we replace the relation " $\in$ " by other relations that lead to nonsense we get a much larger class of contradictions called self-reference. For example, suppose we replace " $\in$ " by "the ball half its size", can anyone comprehend what that ball is? Belonging to this type of paradox are these relations: "the snake that swallowed itself" (let it swallow the tail first) and "the basket inside itself". The indirect proof is self-referent because the conclusion of the theorem being proved rests on the negation of its hypothesis (a theorem, by the way, includes its proof). The indirect proof is based on the principle of the excluded middle of formal logic which is what the Russell antimony is and rejected by intuitionism and the broader philosophy of constructivism that extends to mathematics education where the teacher does not lecture but sets the right conditions for the student to create the appropriate concepts for the situation [31]-[35].

Gödel's second incompleteness theorem states that no consistent axiomatic system which includes Peano arithmetic (Peano's axioms) can prove its own consistency. This can be stated simply and in more general terms: No consistent axiomatic system (mathematical space) can prove its own consistency. This theorem is true because a proof would be self-referent.

\section{The Status of Set Theory}

The creator of set theory was Georg Cantor [20] [21] who introduced, among others, the concepts infinity, cardinal number and the axiom of choice. The subject was revisited by Russell and Whitehead who developed set theory on the axioms of formal logic in their joint work, Principia Mathematica [36]-[38]. This work attempts to reduce the foundations of mathematics to logic [39] [40]. Although the theory of types in Principia did resolve the Russell paradoxes, it was impossible to say that a class was or was not a member of itself. We have resolved 
this problem by putting the set of all sets where each element does not belong to itself and the set of all sets where each element belongs to itself in the category of vacuous set that must be avoided. Today, the most advanced development of set theory is Zermelo-Fraenkel with the axiom of choice or ZFC [20]. However, both its axioms of infinity and choice bring in uncontained ambiguity to it.

We identify two aspects of set theory: its role (1) as a fundamental language and repository of the basic principles of modern mathematics and (2) as an independent branch of formal or mathematical logic (propositional calculus). The first role does not apply to any other mathematical spaces since every mathematical space is defined solely by its axioms including its rules of inference for proving theorems. In other words, universal rules of inference such as formal logic do not apply to any other mathematical space since they have nothing to do with its axioms. As an independent mathematical space it is also flawed by the inclusion of the axiom of infinity and the axiom of choice. Therefore, the program of logicism to develop a universal language for mathematics failed and whatever concepts of set is needed in mathematics can take refuge in the appropriate mathematical spaces where they are defined.

\section{The Participants in the Debate}

We present briefly the three philosophies of or schools of thought in mathematics.

\subsection{Logicism}

What is the status of logicism? It aims to build mathematics on the axioms and propositions (theorems) of formal logic which is cast in the language of set theory. The axioms of formal logic consists of the laws of classical logic [40] plus type theory [41] that provides the remedy for the Russell paradoxes and, essentially, avoids vacuous concepts such as the set of all sets where each element does not belong to itself from which follows that the universal and power sets of a set are vacuous and do not exist and the relation " $\in$ " ("is a member of") is not reflexive. Moreover, we require that the relation " $\in$ " is neither reflexive nor transitive, i.e., $A \in B$ and $B \in C$ does not imply $\mathrm{A} \in \mathrm{C}$; in other words, there is no conclusion that can be drawn from $\mathrm{A} \in \mathrm{B}$ and $\mathrm{B} \in \mathrm{C}$. In addition, an axiom schema [39] is introduced that generates additional axioms. The rules of inference are defined by these axioms. A proposition is true by virtue of its form derived from the axioms [1]. The aim of logicism is to establish set theory as the universal language of mathematics, i.e., as the language of all mathematical spaces. First, the concepts of a mathematical space are defined in terms of the language of set theory. Then its axioms are derived from set theory in accordance with the rules of inference of formal logic or propositional calculus. Both, of course, are subject to the limitations of set theory. All the three philosophies of mathematics consider the axioms self-evident and true. However, what is self-evident to one may not be so to another and, as we have seen, thought can create contradictory and vacuous concepts, e.g., the complex number $i$. Consequently, there exist Euclidean and non-Euclidean geometry, Abelian and non-Abelian group and Hausdorff and non-Hausdorff mathematical spaces all of which created by thought. This nature of thought is explained in [13] [42] [43].

The natural numbers are defined in the language of set theory by the axioms of Peano [20]:

1) Zero is a number.

2) If $a$ is a number, the successor of $a$ is a number.

3) Zero is not the successor of a number.

4) Two numbers of which the successors are equal are themselves equal.

5) (Induction Axiom) if a set $S$ of numbers contains zero and also the successor of every number in $S$, then every number is in S.

Logicism failed to prove the axioms of arithmetic and its goal of deriving arithmetic from formal logic. Recall that it is the representation of thought in the real world that constitutes a mathematical space and not the concepts of individual thought. Therefore, writing the concepts of a mathematical space in the language of set theory and proving its theorems by the rules of inference of formal logic negates its axioms as the sole determinant of its structure, properties and behavior.

\subsection{Intuitionism}

The rejection of the indirect proof by intuitionism [2] is the foundation of constructivism which has been extended to a philosophy of mathematics-science education now called creative mathematics-science education 
[31]-[35]. Consequently, Brouwer had to reject his earlier important contribution to Analysis and Topology named after him, the Brouwer fixed point theorem [44], because the proof rests on the indirect proof. However, constructivism as a philosophy is more than simply the rejection of the indirect proof and extension to mathematics-science-education since as we have seen it has engendered a new philosophy of education. Like logicism and formalism, intuitionism correctly views concepts as creation of thought but incorrectly assumes that human thought is invariant or the same among individuals. This is not the case according to [13] [42] [43]. In fact, individual thought is conditioned or shaped by the cultural milieu and one's training and experience. Moreover, we recall that individual thought is capable of creating ambiguous, vacuous and contradictory concepts. Constructivism is a modification of the Socratic method of teaching where the student states his opinion on a subject or an answer to a question and the teacher asks a series of leading questions towards the correct answer [33]-[35]. In constructivist teaching the teacher provides the condition in the classroom so that the student creates the appropriate concepts for the situation. Moreover, intuitionism correctly rejects concept or solution that cannot be constructed. Therefore, the solution must be determined by actual construction but the latter preceded by proof of its existence.

\subsection{Formalism}

Formalism recognizes the ambiguity of the concepts of individual thought since it is not accessible to others and cannot be discussed collectively [3]. Therefore, it requires concepts to be objects in the real world that can be collectively observed such as words, symbols, figures and chess pieces. In a chess game the axioms are the rules of the game. In other words, the subject matter of mathematics cannot be the concepts of individual thought but objects in the real world (also called concepts) subject to the axioms of the given mathematical space. Both logicism and intuitionism hold that an axiom is self-evident by virtue of its form regardless of content [1] [2]; intuitionism says that a concept or an axiom is "evident from profound intuitionist reflection" [2]. At the same time, intuitionism admits correct ideas based on intuition but limits them to those that can be constructed or actualized which is consistent with the rejection of the principle of excluded middle, the basis of the indirect proof and a contradiction. Formalism does not challenge the logicist view but retains the indirect proof [3]. This contradicts the formalist requirement that the concepts and propositions of a mathematical space be subject to consistent axioms. Among the implications of this requirement are: 1) mathematical truth is not absolute but relative to the axioms of a mathematical space. In other words, when we say that a theorem is true it is relative to the mathematical space where the theorem belongs. A theorem in our sense is a sequence of arguments or propositions from the hypothesis to the conclusion, where each proposition except the hypothesis follows from the preceding proposition; 2) to avoid ambiguity every concept of a mathematical space must be defined by its axioms and the choice of the axioms is not complete until every concept is defined, i.e., undefined terms are inadmissible. 3) The rules of inference must follow from the axioms. 4) Therefore, as noted earlier, universalrulesofinferenceincludingformallogicdonotapplytoanyothermathematicalspace. 5) Consequently, there cannot be a single set of axioms that defines traditional mathematics (which is the goal of logicism) or even geometry since there are contradictory mathematical spaces, e.g., Euclidean and Non-Euclidean geometry and Archimedean and non-Archimedean number systems. 6) The goal of logicism which is to derive arithmetic from formal logic is not achievable because formal logic has its own independent set of axioms.

Intuition still plays a role in mathematics based on experience but needs verification by the axioms or experience. For example, a skillful chess player takes the next move based on intuition (that comes with experience) and the position of the pieces; in this case, however, he cannot prove the correctness of his next move from the rules of the game due to time limitation. In this regard, we pose an open problem: Is there a position of a player's pieces that insures a win when attained in the course of a game?

The main contribution of logicism is the discovery of the Russell paradoxes and the remedy by type theory but left unresolved the flaw in set theory and contradiction in self-reference. It also failed to derive arithmetic from set theory. The main contribution of intuitionism is constructivist mathematics with the rejection of the indirect proof and the requirement that concepts and propositions be constructible, that of formalism the representation of thought by objects in the real world subject to consistent axioms. All three schools of thought agree that concepts are created by thought but do not distinguish between abstract concept, i.e., concept that has no referent in the real world such as mathematical concepts, and physical concept, i.e., concept that has referent in the real world such as atom, star and our universe. Moreover, formalism retains the indirect proof and like intuitionism 
does not openly challenge the logicist proof of theorems. However, in practice the formalist proves theorems directly from the axioms. Technical mathematics, especially, those with applications to science is constructivist. Non-constructivist applications of mathematics are unreliable and could be dangerous as what happened to the disastrous final flight of the Columbia Space Shuttle in 2004 [45] [46].

\section{The New Foundations of Mathematics}

The resolution of the great debate which includes the critique-rectification of the foundations of traditional mathematics plus new requirements on every mathematical space to avoid uncontained ambiguity constitutes the new foundations of mathematics. The requirements include what axioms to choose that define a mathematical space. Our aim is to choose axioms that serve science and its applications, e.g., natural science, engineering, medicine and social science. The new requirements are:

1) Constructivism which requires the new mathematical methodology, qualitative mathematics. Among the earliest constructivist mathematical space is the rectified calculus of variations together with functional analysis and optimal control theory spurred by the discovery of the generalized curves and surfaces by L. C. Young that solved the calculus of variations and optimal control problems on curves and surfaces starting in 1937 [47]-[52]. Incidentally, this is also the constructivist solution of Problem 23 of Hilbert's 23 problems [20] posed a century ago. The most recent constructivist mathematics is the mathematics of GUT [53] that includes the new real number system and complex vector plane, nested generalized physical fractal and generalized integral and derivative [16] [17] [54]-[57].

2) Admission of concepts only when defined solely by the axioms; this is a departure from traditional mathematics that admits undefined terms or concepts other than grammatical connectives which have no bearing on thought. The choice of the axioms of a mathematical space is not complete until every concept is defined; when this phase is completed the mathematical space becomes deductive and every theorem follows from the axioms.

3) Avoidance of uncontained ambiguity.

4) Anchoring the rules of inference on the axioms.

5) Enhancing accuracy, clarity and simplicity by defining the relation " $=$ " to mean "the same as" or "equivalent to" so that it is an equivalence relation and satisfies the identity, reflexivity, and transitivity properties which need not be taken as axioms.

At this point we focus on the real number system which lies at the foundations of mathematics. We have already noted the inconsistency arising from the ambiguity of the axiom of choice one of its defining field axioms. Then in the early 1920s Brouwer constructed a counterexample to the trichotomy axiom, another Field axiom [58]. In 1992 the author constructed a counterexample to it ([16]) independently which showed at the same time that the real number system is not linearly ordered; the latter collapses not only the real number system but also real and complex analysis. The counterexample is a theorem in [16] [17] [30] [57]. These inconsistences of the real number system are the rationale for its reconstruction into the consistent new real number system which provided the counterexample to FLT that proved it false [16]. The counterexample has since then been extended to countably infinite counterexamples in [16]-[18] [30] [57] [59].

The axioms of the new real number system $\mathbf{R}^{*}$ are: Axiom $1: 0,1 \in \mathbf{R}^{*}$ where 0 and 1 are the additive and multiplicative identities defined by the addition and multiplication tables, respectively; Axiom 2: the addition table, and Axiom 3: the multiplication table. The identity elements 0 and 1, are defined by the addition and multiplication tables of Axioms 2 and 3; so are the rest of the integers which are the successors of $0,1,2, \cdots$, (by integers we mean their representation in the real world since the concepts of thought are ambiguous; at present we use the Hindu-Arabic numerals for the integers and the metric system for the real numbers). Then in the definition of the real numbers (decimals) in [16] the integers are formally defined as the integral parts of the decimals. Then for the first time the isomorphism between the natural numbers and the integers was established defining at the same time that the natural numbers are the integers. Moreover, the addition to the identity, reflexivity and transitivity properties of "=”, the commutativity and associativity of addition and multiplication and the distributivity of multiplication with respect to addition follow from the addition and multiplication tables and need not be taken as additional axioms for the real number system. How do we interpret $2+3=5$ ? Addition is a binary mapping or operation $f_{+}$on $\mathbf{R}^{*}$ so that $2+3=f_{+}(2,3)=5$, the image of $(2,3)$ under $f_{+}$in accordance with the addition table. This shuts off the controversy in cyberspace around the equation $1=0.99 \cdots$ which raged for over a decade since 1998 . The equation is wrong because 1 and $0.99 \cdots$ are distinct objects; it is akin to the equation 
orange $=$ apple. In fact, in the new real number system, $0.99 \cdots<1$ and $1-0.99 \cdots=$ the dark number $\mathrm{d}^{*}$ which is not a decimal but an element of $\mathbf{R}^{*}$.

Consider the paradoxes (contradictions) that we have identified. The Russell paradoxes involve relations that are reducible to the relation $\epsilon=$ "is a member of" or $\notin=$ "is not a member of" which have been resolved by Russell's theory of types that orders propositions into a linear hierarchy of orders [41]. Then the power set of a nonempty set A is inadmissible for it would lead to the Russell antimony $\mathrm{m} \in \mathrm{m}$; so is the universal set. The resolution of self-reference is simply to avoid it. If we eliminate self-referent propositions the indirect proof collapses since its conclusion rests on the negation of the hypothesis, a case of self-reference. Thus, the resolution of the Great Debate is constructivist and meets the other requirements we have set to avoid uncontained ambiguity (Constructivist mathematics is the subject of a sequel to this paper which will be illustrated in the constructivist development of $\mathbf{R}^{*}$, the g-closure of $\mathbf{R}$ [16] [17]).

Finally, we include in the new foundations of mathematics the rectification work on the real number system $\mathbf{R}$ and complex number system $\mathbf{C}$ which is the replacement of the field axioms by the axioms of the new real number system and the replacement of the complex number $i$ by the plane vector operator $\mathbf{j}$ :

Axiom 1. $\mathbf{R}^{*}$ contains the elements 0,1 .

Axiom 2. The addition table (Table 1).

Axiom 3. The multiplication table (Table 2).

The partial Table 1 and Table 2 below are axioms because they insure the existence of the integers, define addition and multiplication as binary operations on them and specify their properties. We first construct the

Table 1 . The addition table.

\begin{tabular}{|c|c|c|c|c|c|c|c|c|c|c|}
\hline+ & 0 & 1 & 2 & 3 & 4 & 5 & 6 & 7 & 8 & 9 \\
\hline 0 & 0 & 1 & 2 & 3 & 4 & 5 & 6 & 7 & 8 & 9 \\
\hline 1 & 1 & 2 & 3 & 4 & 5 & 6 & 7 & 8 & 9 & \\
\hline 2 & 2 & 3 & 4 & 5 & 6 & 7 & 8 & 9 & & \\
\hline 3 & 3 & 4 & 5 & 6 & 7 & 8 & 9 & & & \\
\hline 4 & 4 & 5 & 6 & 7 & 8 & 9 & & & & \\
\hline 5 & 5 & 6 & 7 & 8 & 9 & & & & & \\
\hline 6 & 6 & 7 & 8 & 9 & & & & & & \\
\hline 7 & 7 & 8 & 9 & & & & & & & \\
\hline 8 & 8 & 9 & & & & & & & & \\
\hline 9 & 9 & & & & & & & & & \\
\hline
\end{tabular}

Table 2. The multiplication table.

\begin{tabular}{|c|c|c|c|c|c|c|c|c|c|c|}
\hline$\times$ & 0 & 1 & 2 & 3 & 4 & 5 & 6 & 7 & 8 & 9 \\
\hline 0 & 0 & 0 & 0 & 0 & 0 & 0 & 0 & 0 & 0 & 0 \\
\hline 1 & 0 & 1 & 2 & 3 & 4 & 5 & 6 & 7 & 8 & 9 \\
\hline 2 & 0 & 2 & 4 & 6 & 8 & & & & & \\
\hline 3 & 0 & 3 & 6 & 9 & & & & & & \\
\hline 4 & 0 & 4 & 8 & & & & & & & \\
\hline 5 & 0 & 5 & & & & & & & & \\
\hline 6 & 0 & 6 & & & & & & & & \\
\hline 7 & 0 & 7 & & & & & & & & \\
\hline 8 & 0 & 8 & & & & & & & & \\
\hline 9 & 0 & 9 & & & & & & & & \\
\hline
\end{tabular}


digits or basic integers: $2=1+1,3=2+1,4=3+1,5=4+1,6=5+1,7=6+1,8=7+1,9=8+1$ which apply only to the integers which are sums and products. It is also the metric system of numeration or scientific notation for large numbers. To this end, we define the base integer $10=1+9$.

Their extension to the full tables using the Hindu Arabic or base 10 numerals is quite familiar and will not concern us here.

Since the tables define finite sums and products the laws of addition and multiplication of arithmetic can be verified from them. In either case, finite mathematical induction can be applied if needed. The system of Hindu-Arabic numerals quantitatively models the metric system as a system of measurement.

Our proposed remedy for the vacuous concept $i$ of the complex number system $\mathbf{C}$ is its replacement by the operator $\mathbf{j}$ defined as left-right operator on or mapping of a plane vector by positive (counterclockwise) rotation about the origin by $\pi / 2$. Then the coordinate axes are: the $\mathrm{x}$-axis $(\mathrm{x}, 0)$, application of $\mathbf{j}$ on the $\mathrm{x}$-axis (rotation of $(\mathrm{x}, 0)$ by $\pi / 2)$, to obtain the $\mathrm{y}$-axis, i.e., $\mathbf{j}(\mathrm{x}, 0)=\mathbf{j y}$; application of $\mathbf{j}$ on $\mathrm{y}$-axis (rotation of the $\mathrm{y}$-axis by $\pi / 2)$ to obtain the $-\mathrm{x}$-axis, i.e., $\mathbf{j j}(\mathrm{x}, 0)=-(\mathrm{x}, 0)=(-\mathrm{x}, 0)$; application of $\mathbf{j}$ on the $-\mathrm{x}$-axis (rotation by $\pi / 2)$ to obtain the $-\mathrm{y}$-axis; , i.e., $\mathbf{j}(-\mathrm{x}, 0)=-\mathbf{j y}$ and application of $\mathbf{j}$ on the $-\mathrm{y}$-axis (rotation by $\pi / 2)$ to obtain the $\mathrm{x}-\mathrm{axis}$, i.e., $\mathbf{j}(-\mathrm{y}, 0)$ $=(\mathrm{x}, 0)$. Thus, we have the cyclic values of the composites of $\mathbf{j}$ with itself:

$$
\mathbf{j}(\mathbf{1})=\mathbf{j}, \mathbf{j} \mathbf{j}(\mathbf{1})=\mathbf{j}^{2}=-\mathbf{1}, \mathbf{j j}^{2}(\mathbf{1})=\mathbf{j}^{3}=-\mathbf{j}, \mathbf{j}\left(\mathbf{j}^{3}\right)=\mathbf{j}^{4}=\mathbf{1}
$$

where 1 is the unit vector along the x-axis.

They are the vector basis of the complex vector plane [10]. We define the operator $-\mathbf{j}$ as inverse operator of $\mathbf{j}$, i.e., when applied on a vector $\mathbf{v}$ we rotate it clockwise through $\pi / 2$. Note: $-\mathbf{j}(\mathbf{1})=\mathbf{j}(-\mathbf{1})$. Applying composite mappings on the unit vector $\mathbf{1}$ along the $\mathrm{x}$-axis successively, we have the four cyclic images of $\mathbf{1}$ in (2) in reverse order. For $n>4$, the cycle is repeated and we obtain $\mathbf{j}^{n}=\mathbf{j}\left(\mathbf{j}^{n-1}\right), n=1,2, \cdots$, where we define $\mathbf{j}^{0}=\mathbf{1}$. Then by replacing both the $\mathrm{x}$-axis and yi axis by the new real number system and yj, where y ranges through the new real numbers, we obtain the complex vector plane $C^{*}$, the full rectification of the complex number system [17]. The constructivist development of the new real number system and complex vector plane will be the content of a sequel to this paper.

\section{Status of Hilbert's Problems and Other Long-Standing Problems of Mathematics}

Hilbert's problems originally consist of unsolved problems in mathematics proposed by Hilbert. Ten of them were presented at the Second International Congress of Mathematicians in Paris on August 8, 1900. They were problems $1,2,6,7,8,13,16,19,21$, and 22. The complete list is in [9]. They were designed to serve as examples of the kinds of problems the solutions of which would advance mathematics. As such, some were areas for investigation not strictly “problems”. Reference [60] lists over a hundred problems including some of Hilbert’s problems that have been solved but we focus on Hilbert's problems.

1) "Cantor's problem of the cardinal number of the continuum." The question of whether there is a transfinite number between that of a denumerable set and the numbers of the continuum was answered by Gödel and Cohen in their solution to the continuum hypothesis to the effect that the answer depends on the particular version of set theory assumed. The question of whether the continuum of numbers should be considered a well ordered set is related to Zermelo's axiom of choice. In 1963, the axiom of choice was demonstrated to be independent of all other axioms of set theory. There is universal consensus on whether these results give a solution to this problem. (Of course, mathematics is not a matter of consensus)

2) "The compatibility of the arithmetical axioms.” Gödel's second incompleteness theorem indicated that it cannot be proven that the axioms of logic are consistent in the sense that any formal system interesting enough to formulate its own consistency can prove its own consistency if it is inconsistent. There is no consensus if the results of Gödel and Gentzen provide a solution.

3) Give two tetrahedra that cannot be decomposed into congruent tetrahedra directly or by adjoining congruent tetrahedra. Dehn $(1900,1902)$ showed that a regular tetrahedron cannot be decomposed into a finite number of congruent tetrahedra (directly or by joining congruent tetrahedra) which can be reassembled to make a cube. It follows immediately from this result that two tetrahedra cannot be decomposed, as Hilbert proposed.

4) Find geometries whose axioms are closest to those of Euclidean geometry if the ordering and incidence axioms are retained, the congruence axioms weakened, and the equivalent of the parallel postulate omitted. This problem was solved by G. Hamel. 
5) Can the assumption of differentiability for functions defining a continuous transformation group be avoided? This is a generalization of the Cauchy functional equation solved by John von Neumann in 1930 for bicompact groups. Also solved for the Abelian case, and for the solvable case in 1952 with complementary results by Montgomery and Zippin (subsequently combined by Yamabe in 1953). Andrew Gleason showed in 1952 that the answer is also "yes” for all locally bicompact groups.

6) Can physics be axiomatized?

7) Let $\alpha \neq 1 \neq 0$ be algebraic and $\beta$ irrational. Is $\alpha^{\beta}$ transcendental? In particular, are the Gelfond-Schneider constant $2^{\sqrt{2}}$ and Gelfond's constant $\mathrm{e}^{\pi}$ transcendental (Wells, 1986)? A $\beta$ is known to be transcendental for the special case of an irrational algebraic number, as proved in 1934 by Aleksander Gelfond in a result now known as Gelfond's theorem (Courant and Robins, 1996). However, the case of nonalgebraic irrational $\beta$ has not been resolved, with solutions known only for degenerate constructions such as $\alpha=2, \beta=\ln 3 / \ln 2$.

8) Prove the Riemann hypothesis. The conjecture has been neither proved nor disproved.

9) Construct generalizations of the reciprocity theorem of number theory.

10) Does there exist a universal algorithm for solving Diophantine equations? The impossibility of obtaining a general solution was proven by Yuri Matiyasevich in 1970 (Matiyasevich, 1970; Davis, 1973; Davis and Hersh, 1973; Davis, 1982; Matiyasevich, 1993) by showing that the relation $n=F_{2 m}$ (where is the (2 m) ${ }^{\text {Th }}$ Fibonacci number) is Diophantine. More specifically, Matiyasevich showed that there is a polynomial Pin $n, m$, and a number of other variables $x, y, z, \cdots$ having the property that $n=F_{2 m}$ iff there exist integers $x, y, z, \cdots$ such that $P(n$, $m, x, y, z)=0$.

11) Extend the results obtained for quadratic fields to arbitrary integer algebraic fields.

12) Extend a theorem of Kronecker to arbitrary algebraic fields by explicitly constructing Hilbert class fields using special values. This calls for the construction of holomorphic functions in several variables which have properties analogous to the exponential function and elliptic modular functions (Holzapfel, 1995).

13) Show the impossibility of solving the general seventh degree equation by functions of two variables.

14) Show the finiteness of systems of relatively integral functions.

15) Justify Schubert's enumerative geometry (Bell, 1945).

16) Study the topology of real algebraic curves and surfaces. See Gudkov and Utkin (1978), Ilyashenko and Yakovenko (1995), and Smale (2000) for additional details.

17) Find a representation of definite form by squares.

18) Build spaces with congruent polyhedra.

19) Analyze the analytic character of solutions to variational problems.

20) Solve general boundary value problems.

21) Solve differential equations given a monodromy group. More technically, prove that there always exists a Fuchsian system with given singularities and a given monodromy group. Several special cases had been solved, but a negative solution was found in 1989 by B. Bolibruch (Anasov and Bolibruch, 1994).

22) Uniformization.

23) Extend the methods of calculus of variations.

\section{Assessment of Hilbert's Problems}

We mark by an asterisk (*) when the solution of a problem has been challenged or replaced by the author, by double asterisk (**) when the solution of a problem has not been reviewed by the author and by triple asterisk $(* * *)$ when a problem has been solved but not listed among the solved problems. An unmarked problem is open.

1)* Problem 1 was supposedly solved by Gödel and Cohen in their proof of the continuum hypothesis [23] [24] according to [9]. There are two reasons why the proof is flawed: (1) Cantor's proof that the reals are nondenumerable is flawed because it involves the application of the axiom of choice on infinite set and (2) the power set $\mathrm{S}$ of a denumerable set does not exist being a Russell antimony type paradox that leads to the conclusion $\mathrm{S}$ $\in \mathrm{S}$ and $\mathrm{S} \notin \mathrm{S}$. Therefore, the problem is vacuous and has no solution. Moreover, the discussion of the solution confirms our position that the language of set theory cannot be a universal language for mathematics because the arguments and conclusions vary with the kind of or axioms for the set theory being applied.

2)* Problem 2 is incorrectly formulated and we replace it by a general theorem: Any mathematical space (consistent axiom system) cannot prove its own consistency or inconsistency for such a proof would be self-referent and flawed. Consensus is inadmissible in constructivist mathematics being a deductive system; a theorem 
or solution to a problem follows from the axioms.

$3)^{* *}$ It is reported in [9] that the solution follows from a result by Dehn $(1900,1902)$.

4)** It is reported in [9] that Problem 4 has been solved by G. Hamel.

$5)^{* *}$ The problem has been solved for special cases [9].

6)*** The answer to problem 6 is yes and the author provided the solution in 2008, namely, the Grand Unified Theory (GUT) [10]. The solution is constructivist.

7)* Problem 7 has been partially solved according to [9] but based on the new foundations of mathematics the problem itself is ambiguous because it involves the ill-defined irrational and, naturally, the supposed partial solution is flawed.

8) Problems 8 and 9 are open.

10)** We have seen that the real number system is not well defined because two of its axioms, the axiom of choice or its variants - the Dedekind cut and completeness_-are ambiguous and false on infinite set. At the same time, the claim of impossibility of obtaining a general solution by Yuri Matiyasevich, Davis and Hershis are not true for the same reason.

11) Problems 11 to 15 are open.

16)** Gudkov and Utkin (1978), Ilyashenko and Yakovenko (1995) and Smale (2000) have done what Problem 16 asks for according to [9].

17) Problems 17 to 20 are open.

21)** Several special cases of Problem 21 had been solved, but a negative solution was found in 1989 by B. Bolibruch (Anasov and Bolibruch, 1994) according to [9].

22) Candidly, the author does not understand Problem 22.

23)*** L. C. Young not only extended the methods of the calculus of variations asked in Problem 23 but also introduced a new method that he applied to functional analysis and optimal control theory in $\mathbf{R}^{n}, n \geq 3$, and solved the general calculus of variations and optimal control problems in the mathematical spaces of curves and surfaces [47]-[52]. The method entails enrichment of the domains of curves and surfaces with the introduction of generalized curves and surfaces, respectively, and a suitable norm called Young measure to achieve a complete space in the norm for each space so that the optimal solution is calculated as the limit in this norm of a sequence of simplicial curves or surfaces which is a generalized curve or surface in either case. The solution is constructivist. There is no indication at all in Young's work that he was aware he solve done of Hilbert's problems. His method of enrichment in a suitable norm has become a standard method that the authored rediscovered and used to develop the new real number system. Although Brouwer introduced the idea of constructivist mathematics and proved theorems that are constructivist, it was Young who first developed a whole body of constructivist mathematics in the $20^{\text {th }}$ Century.

The list in [60] erred in not reporting this major development in mathematics from which sprung modern control theory. It also erred in not reporting the solution of Problem 6 among Hilbert's problems as the most recent major development in mathematics.

***The 378-year-old conjecture by Fermat (popularly known as Fermat's last theorem (FLT)) [11] that says, “The Diophantine equation $x^{n}+y^{n}=z^{n}$ has no integer solutions for $n>2, x y z \neq 0$ ”, was supposedly solved by Wiles and Taylor [61] [62] according to [60]. Unfortunately, the solution was not informed by the counterexample to the trichotomy axiom constructed by Brouwer in 1985 [58] and independently by the author in 1998 [16]. The trichotomy axiom is one of the defining axioms of the real number system [9]. The counterexample to it is another proof that the real number system $\mathbf{R}$ is inconsistent. Since FLT is formulated in $\mathbf{R}$, it is also ambiguous and, naturally, has no solution. Consequently, the proof by Wiles and Taylor is false. To resolve FLT it was necessary to reconstruct the real number system $\mathbf{R}$ into the consistent constructivist new real number system $\mathbf{R}^{*}$ and reformulate FLT in it. The construction was started in 1998 [16] with the introduction of the new integers $N .99 \cdots$, and the dark number $d^{*}=N-(N-1) .99 \cdots, 1-0.99 \cdots, N=1, \cdots$, where $N$ is an integer. The element $d^{*}$ is a new real number but not a real number; $0<\mathrm{d}^{*}<\mathrm{x}, \mathrm{x} \in \mathbf{R}$. It is both the qualitative and quantitative model of the superstring, fundamental building block of matter [63]. The first countable counterexamples to FLT (1998 [18] is the solution of the above Diophantine equation with $(x, y, z)=\left((0.99 \cdots) 10^{T}, d^{*}, 10^{T}\right), T=1,2, \cdots$, that clearly satisfies it. The countably infinite solutions are the triples $(k x, k y, k z), k=1,2, \cdots$, which also satisfy Fermat's equation. The existence of such countably infinite solutions is a theorem in [16]-[18] [30] [57] [59]. The full construction of $\mathbf{R}^{*}$ will be published in a sequel, The Constructivist New Real Number System.

***The 273-year old strong Goldbach's conjecture [12] that says, All positive even integers $\geq 4$ can be ex- 
pressed as the sum of two primes, was proved in 2003 [16]. It is a theorem in [17] [30] [57] [59] [63]. The conjecture and its proof are incorrectly excluded from the list of solved problems listed in [60]. The proof is likewise constructivist.

\section{Conclusions}

One thing stands out in this paper: to pursue constructivist mathematics a new mathematical methodology is needed: qualitative mathematics, the qualitative model of rational thought [13] and the complement of computation and measurement, and a suitable norm, in this case, the g-norm [16] [17]. In the work of L. C. Young this translates into the method of enrichment and the Young Measure.

Examples of qualitative mathematics are abstract mathematical spaces and the search for the laws of nature. In science, the appropriate methodology is qualitative mathematics and modeling [13] that explains how nature works its tool qualitative mathematics. It is the main contribution of [14] and was applied to physics for the first time to solve the gravitational n-body problem [64]. Qualitative mathematics and modeling shifts the subject matter of science from the appearances of nature (natural phenomena) to nature itself and how it works and lifts traditional science to the new science [65] articulated by GUT and its theoretical applications [13] [30] [43] [44] [46] [66]-[68]. Through this methodology GUT unites the natural sciences on the natural laws, the common thread being the superstring. It is clear that natural science and mathematics are distinct disciplines: natural science is the study of nature its language mathematics. On the philosophical side: at the micro and super micro scale nothing is certain and deterministic in nature because of the uncertainty of small numbers that manifests itself in the Heisenberg uncertainty principle. Moreover, while at the micro scale the second law of thermodynamics says that a closed system tends towards greater disorder or chaos or zero entropy [69], the opposite is true at the macro scale. For example, from the chaos of the big bang [70] and the cosmic burst [71], our universe has evolved to its present order in accordance with natural laws [66].

\section{References}

[1] Speaks, J. (2007) Russell’s Logicism. http://www3.nd.edu/ jspeaks/courses/2007-8/43904/_HANDOUTS/Russell-logicism.pdf

[2] van Stigt, W.P. (1990) Brouwer’s Intuitionism. http://www.mscs.mu.edu/ wimr/publica/120521_stigt2.pdf

[3] Philosophy of Mathematics: Formalism. http://www.mathematics-in-europe.eu/home/43-information/history-philosophy/84-philosophy-of-mathematics-formali $\underline{\mathrm{sm}}$

[4] Irvine, A.D. Bertrand A. D. Russell. http://www-history.mcs.st-and.ac.uk/Biographies/Russell.html

[5] Whitehead, A.N. http://www.philosophybasics.com/philosophers_whitehead.html

[6] O’Connor, J.J. and Robertson, E.F. Luitzen Egbertus Jan Brouwer. http://www-history.mcs.st-and.ac.uk/Biographies/Brouwer.html

[7] Weir, A. (2011) Formalism in the Philosophy of Mathematics. http://plato.stanford.edu/entries/formalism-mathematics/

[8] Snapper, E. (1979) The Three Crises in Mathematics: Logicism, Intuitionism and Formalism. http://www.maa.org/sites/default/files/images/upload_library/22/Allendoerfer/1980/0025570x.di021111.02p0048m.pdf

[9] Weisstein, E.W. Hilbert's Problems, MathWorld-A Wolfram Web Resource. http://mathworld.wolfram.com/HilbertsProblems.html

[10] Escultura, E E. (2008) The Grand Unified Theory. Nonlinear Analysis, A-Series: TMA, 69, 823 - 831.

[11] Young, L.C. (1980) Mathematicians and Their Times. North-Holland, Amsterdam.

[12] Davies, P.J. and Hersch, R. (1981) The Mathematical Experience. Birkhäuser, Boston.

[13] Escultura, E.E. (2015) Rational Thought, Cognition and Knowledge. International Education Research (Science and Education Centre of North America), 3, 21 - 37. http://www.todayscience.org/IER/article/ier.v3i1p21.pdf http://dx.doi.org/10.12735/ier.v3i1p21

[14] Escultura, E.E. (1970) The Trajectories, Reachable Set, Minimal Levels and Chains of Trajectories of a Control System. PhD Thesis, University of Wisconsin, Wisconsin.

[15] Lakatos, I., Worral, J. and Zahar, E., Eds. (1976) Proofs and Refutations. Cambridge University Press, Cambridge. http://dx.doi.org/10.1017/CBO9781139171472

[16] Escultura, E.E. (2009) The New Real Number System and Discrete Computation and Calculus. Neural, Parallel and 
Scientific Computations, 17, 59-84.

[17] Escultura, E.E. (2013) Critique-Rectification of Mathematics. In: Escultura, E.E., Ed., Qualitative Mathematics and Modeling: Theoretical and Practical Applications, LAP LAMBERT Academic Publishing, Saarbrücken, 77-129.

[18] Escultura, E.E. (1998) Exact Solutions of Fermat's Equation (Definitive Resolution of Fermat's Last Theorem). Journal of Nonlinear Studies, 5, 227-254.

[19] Royden, H.L. (1983) Real Analysis. 3rd Edition, MacMillan, New York.

[20] Weisstein, E.W. Zermelo-Fraenkel Axioms, MathWorld-A Wolfram Web Resource. http://mathworld.wolfram.com/Zermelo-FraenkelAxioms.html

[21] Weisstein, E.W. Cantor Diagonal Method; MathWorld-A Wolfram Web Resource. http://mathworld.wolfram.com/CantorDiagonalMethod.html

[22] 19th Century Mathematics_Cantor. http://www.storyofmathematics.com/19th_cantor.html

[23] Cohen, P.J. (1966) Set Theory and the Continuum Hypothesis. W. A. Benjamin, Amsterdam.

[24] Szudzik, M. and Weisstein, E.W. Continuum Hypothesis, from Math World-A Wolfram Web Resource.. http://mathworld.wolfram.com/ContinuumHypothesis.html

[25] Weston, T. Banach-Tarski Paradox. http://people.math.umass.edu/ weston/oldpapers/banach.pdf

[26] Merchbacher, E. (1970) Quantum Mechanics. 2nd Edition, John Wiley \& Sons, Inc., New York.

[27] Horgan, H. (1993) The Death of Proof. Scientific American, 269, 74-82. http://dx.doi.org/10.1038/scientificamerican1093-92

[28] Russell Antimony, MathWorld—A Wolfram Web Resource. http://mathworld.wolfram.com/RussellsAntinomy.html

[29] Examples of Russell Paradoxes. https://www.google.com.au/search?q=examples+of+Russell+paradoxes\&biw=1366\&bih=631\&tbm=isch\&tbo=u\&sour ce=univ\&sa=X\&ei=Rs1dVZL8OMONmwXNyIKICA\&ved=0CEcQsAQ

[30] Lakshmikantham, V., Escultura, E.E. and Leela, S. (2009) The Hybrid Grand Unified Theory. Atlantis Press, Paris. http://dx.doi.org/10.2991/978-94-91216-23-7

[31] Escultura, E.E. (2012) Creative Mathematics Education. Creative Education, 3, 45-54. http://www.scirp.org/journal/PaperInformation.aspx?PaperID=17266\#.VNMJ6S6um44 http://dx.doi.org/10.4236/ce.2012.31008

[32] Escultura, E.E. (2013) Creative Science Education. In: Escultura, E.E., Ed., Qualitative Mathematics and Modeling: Theoretical and Practical Applications, LAP LAMBERT Academic Publishing, Saarbrücken, 318-322.

[33] Concept to Classroom. http://www.thirteen.org/edonline/concept2class/constructivism/

[34] Mathematics Education, Math World—A Wolfram Web Resource. http://mathforum.org/mathed/constructivism.html

[35] Sandy Chapman. The Socratic Method: Fostering Critical Thinking. http://teaching.colostate.edu/tips/tip.cfm?tipid=53

[36] 20th Century Mathematics—Russell and Whitehead. http://www.storyofmathematics.com/20th_russell.html

[37] Irvine, A. D. Principia Mathematica. http://plato.stanford.edu/entries/principia-mathematica/

[38] Principia Mathematica. http://plato.stanford.edu/entries/principia-mathematica/

[39] Sakharov, A. and Weisstein, E.W. Propositional Calculus, MathWorld—A Wolfram Web Resource. http://mathworld.wolfram.com/PropositionalCalculus.html

[40] Three Laws of Logic. https://school.carm.org/amember/files/demo3/2_logic/3logic.htm

[41] Type Theory, Stanford Encyclopedia of Philosophy. http://plato.stanford.edu/entries/type-theory/\#1

[42] Escultura, E.E. (2012) The Physics of Intelligence. Journal of Education and Learning, 1, 51-64. http://www.ccsenet.org/journal/index.php/jel/article/view/20095/13268 http://dx.doi.org/10.5539/jel.v1n2p51

[43] Escultura, E.E. (2014) Physics of the Mind. The Journal of the Science of Healing Outcomes, 6, 6-15.

[44] Weisstein, E.W. Brouwer Fixed Point Theorem, from Math World—A Wolfram Web Resource. http://mathworld.wolfram.com/BrouwerFixedPointTheorem.html

[45] Escultura, E.E. (2007) The Pillars of the New Physics and Some Updates. Nonlinear Studies, 14, 241-260.

[46] Escultura, E.E. (2013) Columbia’s Disastrous Final Flight. In: Escultura, E.E., Ed., Qualitative Mathematics and Modeling: Theoretical and Practical Applications, LAP LAMBERT Academic Publishing, Saarbrücken, 281-293.

[47] Young, L.C. (1933) Approximations by Polygons. Proceedings of the Royal Society of London A, 141, 325-341. http://dx.doi.org/10.1098/rspa.1933.0121 
[48] Young, L.C. (1937) Generalized Curves and the Existence of an Attained Absolute Minimum in the Calculus of Variations. Comptes Rendus des Séances de la Société des Sciences et des Lettres de Varsovie, 30, 211-234.

[49] Young, L.C. (1969) Lectures on the Calculus of Variations and Optimal Control Theory. W. B. Saunders, Philadelphia.

[50] Young, L.C. (1955) On Generalized Surfaces of Finite Topological Types. Memoirs of the AMS, Philadelphia.

[51] Young, L.C. (1942) Generalized Surfaces in the Calculus of Variations: I. Generalized Lipschitzian Surfaces. Annals of Mathematics, 43, 84-103. http://dx.doi.org/10.2307/1968882

[52] Young, L.C. (1942) Generalized Surfaces in the Calculus of Variations: II. Mean Surfaces and the Theory of the Problem $\iint f(x, y, p, q) d x d y=$ Min. Annals of Mathematics, 43, 530-544. http://dx.doi.org/10.2307/1968809

[53] Escultura, E.E. (2009) The Mathematics of the Grand Unified Theory. Journal of Nonlinear Analysis, A-Series: Theory: Method and Applications, 71, e420-e431. http://dx.doi.org/10.1016/j.na.2008.11.003

[54] Escultura, E. (2013) The Logic and Fundamental Concepts of the Grand Unified Theory. Journal of Modern Physics, 4, 213-222. http://dx.doi.org/10.4236/jmp.2013.48A021

[55] Escultura, E.E. (2011) Extended Geometrical and Generalized Fractals, Chaos and Applications. In: Brennan, K.J., Ed., Handbook on the Classification and Application of Fractals, Nova Publishers, Hauppauge, 1-39. https://www.novapublishers.com/catalog/product_info.php?products_id=23231

[56] Escultura, E.E. (2011) The Generalized Integral and Derivative. In: Escultura, E.E., Ed., Scientific Natural Philosophy, Bentham Science Publishers, Sharjah, 54-140. http://www.benthamscience.com/ebooks/9781608051786/index.htm http://dx.doi.org/10.2174/97816080527071110101

[57] Escultura, E.E. (2008) Extending the Reach of Computation. Journal of Applied Mathematics Letters, 21, $1074-1081$. http://dx.doi.org/10.1016/j.aml.2007.10.027

[58] Benacerraf, P. and Putnam, H. (1985) Philosophy of Mathematics. Cambridge University Press, Cambridge.

[59] Escultura, E.E. (2011) Scientific Natural Philosophy. Bentham Science Publishers. http://dx.doi.org/10.2174/97816080527071110101

[60] Solved Problems, MathWorld-A Wolfram Web Resource. http://mathworld.wolfram.com/topics/SolvedProblems.html

[61] Wiles, A. and Taylor, R. (1995) Modular Elliptic Curves and Fermat's Last Theorem. Annals of Mathematics, 141, 443-551. http://dx.doi.org/10.2307/2118559

[62] Wiles, A. and Taylor, R. (1995) Ring-Theoretic Properties of Certain Hecke Algebras. Annals of Mathematics, 141, 553-572. http://dx.doi.org/10.2307/2118560

[63] Escultura, E. E. (2003) The Flux Theory of Gravitation XVII. The New Mathematics and Physics. Applied Mathematics and Computation, 138, 127-149. http://dx.doi.org/10.1016/S0096-3003(02)00126-1

[64] Escultura, E. E. (1997) The Solution of the Gravitational n-Body Problem. Nonlinear Analysis, Series A: Theory, Methods and Applications, 30, 5021-5032. http://dx.doi.org/10.1016/s0362-546x(96)00133-2

[65] Escultura, E.E. (2014) The Leap from Traditional to the New Science. Nonlinear Studies, 21, 283-291.

[66] Escultura, E.E. (2005) Dynamic Modeling of Chaos and Turbulence. Nonlinear Analysis, A-Series: TMA, 63, e519e532. http://dx.doi.org/10.1016/j.na.2005.02.052

[67] Escultura, E.E. (2013) Chaos, Turbulence and Fractal: Theory and Applications, Open Access. International Journal of Modern Nonlinear Theory and Application, 2, 176-185.

[68] Escultura, E.E. (2001) Turbulence: Theory, Verification and Applications. Journal of Nonlinear Analysis, A-Series: TMA, 47, 5955-5966.

[69] What Is the Second Law of Thermodynamics. http://www.livescience.com/50941-second-law-thermodynamics.html

[70] Escultura, E.E. (2012) The Big Bang and What It Was. In: O’Connell, J.R. and Hale, A.L., Eds., The Big Bang: Theory, Assumptions and Problems, Nova Science Publishers, 61-102. https://www.novapublishers.com/catalog/productinfo.php?productsid=21109

[71] Schilling, G. (1999) Science, Watching the Universe’s Second Biggest Bang. Science, 283, 2013-2014. 